\title{
LOWER BOUNDS FOR SOME FACTORABLE MATRICES
}

\author{
B. E. RHOADES AND PALI SEN
}

Received 8 March 2006; Revised 6 June 2006; Accepted 22 June 2006

We determine the lower bounds for classes of Rhaly matrices, considered as bounded linear operators on $\ell^{p}$. We improve on and provide correct proofs of the results of the first author (1990).

Copyright (c) 2006 Hindawi Publishing Corporation. All rights reserved.

The following conjecture was posed by Axler and Shields. Let $\left\{x_{n}\right\}$ be a monotone decreasing sequence of nonnegative numbers, $C$ the Cesáro matrix of order one. What is the best constant $K$ for which $\|C x\|_{2} \geq K\|x\|_{2}$ for all such sequences $\left\{x_{n}\right\}$ ? Lyons [3] determined that the best constant is $\pi / \sqrt{6}$. This result was extended to $\ell^{p}$ spaces for $p>1$ by Bennett [1]. In [1], Bennett established the following result, where $B\left(\ell^{p}\right)$ denotes the set of bounded linear operators on $\ell^{p}$.

Theorem 1. Let $\left\{x_{n}\right\}$ be a monotone decreasing nonnegative sequence, let $A \in B\left(\ell^{p}\right)$ with nonnegative entries, and $1<p<\infty$. Then

$$
\|A x\|_{p} \geq L\|x\|_{p}
$$

where

$$
L^{p}:=\inf _{r}(r+1)^{-1} \sum_{j=0}^{\infty}\left(\sum_{k=0}^{r} a_{j k}\right)^{p}=\inf _{r} f(r), \quad \text { say. }
$$

For $A=C$, the minimum occurs at $f(0)$, which is the sum of the $p$ th power of the first column of $C$. The proof of the result of Bennett is relatively easy, when contrasted with the task of finding $L^{p}$ for a particular matrix, or class of matrices.

A factorable matrix is a lower triangular matrix whose nonzero entries $a_{n k}$ can be written in the form $a_{n} b_{k}$, where $a_{n}$ depends only on $n$ and $b_{k}$ depends only on $k$. Rhaly [4-6] defined three classes of matrices, all of which are factorable. 
In [8], Rhoades investigated the lower bounds question for the Rhaly matrices, and obtained some partial results. In this paper, we return to the study of the Rhaly matrices, as special cases of factorable matrices. This paper differs from [8] in three important respects. First, one general result is proved, and the theorems of [8] then follow as special cases. Second, the results of [8] are extended to all $p>1$. Third, as an application of the general procedure developed here, we are able to provide a new proof of [7, Theorem 1$]$ as well as to verify the conjecture that, for the weighted mean methods with $p_{n}=(n+1)^{\alpha}$, $\alpha \geq 1, L^{p}=f(0)$.

For any sequence $\left\{x_{n}\right\}$, the forward difference operator $\Delta$ is defined by $\Delta x_{n}=x_{n}-x_{n+1}$, and $\Delta^{m+1} x_{n}=\Delta\left(\Delta^{m} x_{n}\right)$.

Theorem 2. Let $A$ be a factorable matrix with positive entries, row sums $t_{n}$, and $\left\{a_{n}\right\}$ monotone decreasing. Then sufficient conditions for $f(0)=L^{p}$ are that

$$
\begin{gathered}
\Delta y_{r}^{p}<0, \quad \Delta^{2} y_{r}^{p}>0, \\
\Delta^{2}\left(\frac{1}{\Delta y_{r}^{p}}\right) \leq 0,
\end{gathered}
$$

where $y_{r}=t_{r} / a_{r}$,

$$
\begin{gathered}
\lim _{r \rightarrow \infty} \frac{a_{r+1}^{p} \Delta y_{r+1}^{p}}{\Delta^{2} y_{r}^{p}} \geq 0, \\
t_{0}+2 \Delta y_{0}^{p} \sum_{j=1}^{\infty} a_{j}^{p} \leq 0 .
\end{gathered}
$$

Proof. With

$$
\begin{aligned}
t_{n} & =a_{n} \sum_{k=0}^{n} b_{k}, \quad y_{n}=\frac{t_{n}}{a_{n}}, \\
f(r) & =\frac{1}{r+1} \sum_{j=0}^{\infty}\left(\sum_{k=0}^{r} a_{j k}\right)^{p} \\
& =\frac{1}{r+1}\left[\sum_{j=0}^{r}\left(\sum_{k=0}^{j} a_{j k}\right)^{P}+\sum_{j=r+1}^{\infty}\left(\sum_{k=0}^{r} a_{j k}\right)^{p}\right] \\
& =\frac{1}{r+1}\left[\sum_{j=0}^{r} t_{j}^{p}+y_{r}^{p} \sum_{j=r+1}^{\infty} a_{j}^{p}\right], \\
f(r)-f(r+1)= & \frac{1}{(r+1)(r+2)} \sum_{j=0}^{r} t_{j}^{p}-\frac{t_{r+1}^{p}}{r+2}+\frac{y_{r}^{p} a_{r+1}^{p}}{r+1}+\Delta\left(\frac{y_{r}^{p}}{r+1}\right) \sum_{j=r+2}^{\infty} a_{j}^{p} .
\end{aligned}
$$

Note that

$$
-\frac{t_{r+1}^{p}}{r+2}+\frac{y_{r}^{p} a_{r+1}^{p}}{r+1}=\frac{y_{r}^{p} a_{r+1}^{p}}{r+1}-\frac{\left(a_{r+1} y_{r+1}\right)^{p}}{r+2}=a_{r+1}^{p} \Delta\left(\frac{y_{r}^{p}}{r+1}\right) .
$$


Thus

$$
f(r)-f(r+1)=\frac{1}{(r+1)(r+2)} \sum_{j=0}^{r} t_{j}^{p}+\Delta\left(\frac{y_{r}^{p}}{r+1}\right) \sum_{j=r+1}^{\infty} a_{j}^{p} .
$$

Define

$$
\begin{aligned}
g(r) & =(r+1)(r+2)[f(r)-f(r+1)] \\
& =\sum_{j=0}^{r} t_{j}^{p}+(r+1)(r+2) \Delta\left(\frac{y_{r}^{p}}{r+1}\right) \sum_{j=r+2}^{\infty} a_{j}^{p} .
\end{aligned}
$$

Then

$$
\begin{aligned}
g(r)-g(r+1)= & -t_{r+1}^{p}+(r+2)\left[(r+1) \Delta\left(\frac{y_{r}^{p}}{r+1}\right)-(r+3) \Delta\left(\frac{y_{r+1}^{p}}{r+2}\right)\right] \sum_{j=r+2}^{\infty} a_{j}^{p} \\
& +(r+1)(r+2) \Delta\left(\frac{y_{r}^{p}}{r+1}\right) a_{r+1}^{p} .
\end{aligned}
$$

But

$$
\begin{aligned}
(r+1) & \Delta\left(\frac{y_{r}^{p}}{r+1}\right)-(r+3) \Delta\left(\frac{y_{r+1}^{p}}{r+2}\right) \\
= & (r+1)\left[\frac{y_{r}^{p}}{r+1}-\frac{y_{r+1}^{p}}{r+2}\right]-(r+3)\left[\frac{y_{r+1}^{p}}{r+2}-\frac{y_{r+2}^{p}}{r+3}\right] \\
= & y_{r}^{p}-\frac{(r+1) y_{r+1}^{p}}{r+2}-\frac{(r+3) y_{r+1}^{p}}{r+2}+y_{r+2}^{p}=\Delta^{2} y_{r}^{p}, \\
-t_{r+1}^{p} & +(r+1)(r+2) \Delta\left(\frac{y_{r}^{p}}{r+1}\right) a_{r+1}^{p} \\
= & -\left(a_{r+1} y_{r+1}\right)^{p}+(r+1)(r+2)\left(\frac{y_{r}^{p}}{r+1}-\frac{y_{r+1}^{p}}{r+2}\right) a_{r+1}^{p} \\
= & a_{r+1}^{p}\left[-y_{r+1}^{p}+(r+2) y_{r}^{p}-(r+1) y_{r+1}^{p}\right]=a_{r+1}^{p}(r+2) \Delta y r^{p} .
\end{aligned}
$$

Thus

$$
g(r)-g(r+1)=(r+2) a_{r+1}^{p}\left(\Delta y_{r+1}\right)^{p}+(r+2) \Delta^{2} y_{r}^{p} \sum_{j=r+1}^{\infty} a_{j}^{p} .
$$


4 Factorable matrices

Define

$$
\begin{aligned}
h(r) & =\frac{g(r)-g(r+1)}{(r+2) \Delta^{2} y_{r}^{p}}=\frac{a_{r+1}^{p} \Delta y_{r}^{p}}{\Delta^{2} y_{r}^{p}}+\sum_{j=r+2}^{\infty} a_{j}^{p}, \\
h(r)-h(r+1) & =\frac{a_{r+1}^{p} \Delta y_{r}^{p}}{\Delta^{2} y_{r}^{p}}+a_{r+2}^{p}\left(1-\frac{\Delta y_{r+1}^{p}}{\Delta^{2} y_{r+1}^{p}}\right) \\
& =\frac{1}{\Delta^{2} y_{r}^{p} \Delta^{2} y_{r+1}^{p}}\left[a_{r+1}^{p} \Delta^{2} y_{r+1}^{p} \Delta y_{r}^{p}+a_{r+2}^{p} \Delta^{2} y_{r}^{p}\left(-\Delta y_{r+2}^{p}\right)\right] \\
& =\frac{\Delta y_{r}^{p} \Delta y_{r+2}^{p}}{\Delta^{2} y_{r}^{p} \Delta^{2} y_{r+1}^{p}}\left[\frac{a_{r+1}^{p} \Delta^{2} y_{r+1}^{p}}{\Delta y_{r+2}^{p}}-a_{r+2}^{p}\left(1-\frac{\Delta y_{r+1}^{p}}{\Delta y_{r}^{p}}\right)\right],
\end{aligned}
$$

and $h(r)-h(r+1) \geq 0$ if and only if

$$
a_{r+1}^{p}\left(\frac{\Delta y_{r+1}^{p}}{\Delta y_{r+2}^{p}}-1\right)-a_{r+2}^{p}\left(1-\frac{\Delta y_{r+1}^{p}}{\Delta y_{r}^{p}}\right) \geq 0 .
$$

Since $\left\{a_{r}\right\}$ is monotone decreasing, it is sufficient to have

$$
\frac{\Delta y_{r+1}^{p}}{\Delta y_{r+2}^{p}}-1 \geq 1-\frac{\Delta y_{r+1}^{p}}{\Delta y_{r}^{p}}
$$

that is,

$$
\Delta y_{r+1}^{p}\left(\frac{1}{\Delta y_{r}^{p}}+\frac{1}{\Delta y_{r+2}^{p}}\right) \geq 2 .
$$

Using (3), $\Delta y_{r+1}^{p}>\Delta y_{r+2}^{p}$ and $\Delta y_{r+1}^{p}<\Delta y_{r}^{p}$. Since $\Delta y_{r}^{p}<0$, the above inequality is equivalent to (4). Thus $h$ is monotone decreasing in $r$. From (5), $h$ is nonnegative, so $g$ is monotone decreasing in $r$. From (6), $g(0)$ is negative, so that $f$ is monotone increasing in $r$.

Lemma 3. Define sequences $\{u(r)\}$ and $\{v(r)\}$ by $u(r)=1 / \Delta v(r)$. Then $\Delta^{2} u(r)$ can be written in the form

$$
\Delta^{2} u(r)=\frac{1}{\Delta v(r)}\left[\frac{2 \Delta^{2} v(r) \Delta^{2} v(r+1)}{\Delta v(r+1) \Delta v(r+2)}-\frac{\Delta^{3} v(r)}{\Delta v(r+2)}\right]
$$

Proof. The equation $u(r)=1 / \Delta v(r)$ implies that

$$
\Delta u(r) \Delta v(r)+u(r+1) \Delta^{2} v(r)=0,
$$




$$
\Delta u(r)=-\frac{u(r+1) \Delta^{2} v(r)}{\Delta v(r)}
$$

Hence

$$
\Delta^{2} u(r) \Delta v(r)+2 \Delta u(r+1) \Delta^{2} v(r)+u(r+2) \Delta^{3} v(r)=0
$$

or

$$
\Delta^{2} u(r)=\frac{1}{\Delta v(r)}\left[-2 \Delta^{2} v(r)\left(-\frac{u(r+2) \Delta^{2} v(r+2)}{\Delta v(r+1)}\right)-\frac{\Delta^{3} v(r)}{\Delta v(r+2)}\right]
$$

Lemma 4. Suppose that $v \in C^{3}[0, \infty)$. If, for all $0<t<1, p>1$, one has
(a) $v^{\prime}>0$,
(b) $v^{\prime \prime}>0$
(c) $2\left(v^{\prime \prime}\right)^{2}-v^{\prime} v^{\prime \prime \prime}>0$,

then $\Delta^{2} v(r) \leq 0$.

Proof. Conditions (a) and (b) imply that $\Delta v(r)<0$ and $\Delta^{2} v(r)>0$. Therefore, from (18), $\Delta^{2} u(r) \leq 0$.

The Rhaly generalized Cesáro matrices [4] are factorable matrices with nonzero entries $a_{n}=t^{n} /(n+1), b_{k}=t^{-k}$, where $0<t<1$. If $t=1$, the matrix reduces to $C$.

Theorem 5. Let $p>1$. Then, for the Rhaly generalized Cesáro matrices, $L^{p}=f(0)$ for $t_{0} \leq$ $t<1$, where $t_{0}$ satisfies

$$
1-2\left[\left(\frac{1+t_{0}}{t_{0}}\right)^{p}-1\right] \sum_{j=1}^{\infty}\left(\frac{t_{0}^{j}}{j+1}\right)^{p}=0
$$

Proof. First, we will show that conditions (a)-(c) of Lemma 4 are satisfied.

Clearly

$$
t_{n}=\frac{1-t^{n+1}}{(n+1)(1-t)}, \quad y_{n}=\frac{1-t^{n+1}}{t^{n}(1-t)}
$$

Thus

$$
\begin{aligned}
v(r) & =\frac{1}{(1-t)^{p}}\left(\frac{1-t^{r+1}}{t^{r}}\right)^{p}, \\
v^{\prime}(r) & =\frac{p t^{-r} \log (1 / t)}{(1-t)^{p}}\left(t^{-r}-t\right)^{p-1}, \\
v^{\prime \prime}(r) & =p(1-t)^{-p}\left(t^{-r}-t\right)^{p-2}\left(p t^{-2 r}-t^{-r+1}\right)\left(\log \left(\frac{1}{t}\right)\right)^{2},
\end{aligned}
$$


6 Factorable matrices

and (a) and (b) are satisfied.

$$
\begin{aligned}
& v^{\prime \prime \prime}(r)=p(1-t)^{-p}\left(\log \left(\frac{1}{t}\right)\right)^{2}\left[(p-2)\left(t^{-r}-t\right)^{p-3}\left(-t^{-r} \log t\right)\left(p t^{-2 r}-t^{-r+1}\right)\right. \\
& \left.+\left(t^{-r}-t\right)^{p-2}\left(-2 p t^{-2 r} \log t+t^{-r+1} \log t\right)\right] \\
& =p(1-t)^{-p}\left(t^{-r}-t\right)^{p-3} t^{-3 r}\left(\log \left(\frac{1}{t}\right)\right)^{3} \\
& \times\left[(p-2)\left(p-t^{r+1}\right)+\left(t^{-r}-t\right)\left(2 p t^{r}-t^{2 r+1}\right)\right] .
\end{aligned}
$$

It then follows that

$$
\begin{aligned}
2\left(v^{\prime \prime}\right)^{2}-v^{\prime} v^{\prime \prime \prime}= & 2\left[p(1-t)^{-p}\left(t^{-r}-t\right)^{p-2}\left(p t^{-2 r}-t^{-r+1}\right)\left(\log \left(\frac{1}{t}\right)\right)^{2}\right]^{2} \\
& -p(1-t)^{-p} t^{-r} \log \left(\frac{1}{t}\right)\left(t^{-r}-t\right)^{p-1} \\
& \times p(1-t)^{-p}\left(t^{-r}-t\right)^{p-3} t^{-3 r}\left(\log \left(\frac{1}{t}\right)\right)^{3} \\
& \times\left[p^{2}-(3 p-1) t^{r+1}+t^{2 r+2}\right] \\
= & p^{2} t^{-4 r}(1-t)^{-2 p}\left(\log \left(\frac{1}{t}\right)\right)^{4}\left(t^{-r}-t\right)^{2 p-4} w(r, p)
\end{aligned}
$$

where

$$
w(r)=p^{2}-(p+1) t^{r+1}+t^{2 r+2} .
$$

Note that $w^{\prime}(r)>0$ for $p \geq 1$. Therefore, $w$ is monotone increasing in $r$. Since $w(0)>$ $0, w$ is positive for $0<t<1, r \geq 0$, and condition (4) is satisfied. Thus $h$ is monotone decreasing in $r$ :

$$
\begin{aligned}
\lim _{r \rightarrow \infty} h(r) & =\lim _{r \rightarrow \infty}\left(\frac{t^{r+1}}{r+2}\right)^{p} \\
& =\frac{\left[\left(1 /(1-t)^{p}\right)\left\{\left(1-t^{r+2} / t^{r+1}\right)^{p}-\left(1-t^{r+3} / t^{r+2}\right)^{p}\right\}\right]}{\left[\left(1 /(1-t)^{p}\right)\left\{\left(1-t^{r+1} / t^{r}\right)^{p}-2\left(1-t^{r+2} / t^{r+1}\right)^{p}+\left(1-t^{r+3} / t^{r+2}\right)^{p}\right\}\right]} .
\end{aligned}
$$


Since the limit of the quantity in brackets is $\left(t^{p}-1\right) /\left(t^{p}-1\right)^{2}, \lim _{r \rightarrow \infty} h(r)=0$, and condition (5) of Theorem 2 is satisfied; that is, $g$ is monotone decreasing in $p$,

$$
\begin{aligned}
g(0)= & t_{0}^{p}+2\left[y_{0}^{p}-y_{1}^{p}\right] \sum_{j=1}^{\infty} a_{j}^{p} \\
= & 1-2\left[\left(\frac{1+t}{t}\right)^{p}-1\right] \sum_{j=1}^{\infty}\left(\frac{t^{j}}{j+1}\right)^{p}=q(t, p), \quad \text { say, } \\
\frac{\partial q}{\partial t}= & -2 p\left(\frac{1+t}{t}\right)^{p-1}\left(-\frac{1}{t^{2}}\right) \sum_{j=1}^{\infty}\left(\frac{t^{j}}{j+1}\right)^{p} \\
& -2\left[\left(\frac{1+t}{t}\right)^{p}-1 p\right] \sum_{j=1}^{\infty} \frac{p j t^{j-1}}{(j+1)^{p}} \\
= & 2 p \sum_{j=1}^{\infty}\left(\frac{t^{j}}{j+1}\right)^{p}\left[\left(\frac{1+t}{t}\right)^{p-1} \frac{1}{t^{2}}-\left(\left(\frac{1+t}{t}\right)^{p}-1\right) \frac{j}{t}\right] .
\end{aligned}
$$

Since the coefficient of $j$ is negative, the quantity in brackets is monotone decreasing in $j$. For $j=1$, it becomes

$$
\begin{aligned}
& \frac{1}{t^{2}}\left[\left(\frac{1+t}{t}\right)^{p-1}+t-t\left(\frac{1+t}{t}\right)^{p}\right] \\
& \quad=\frac{1}{t^{2}}\left[\left(\frac{1+t}{t}\right)^{p-1}(1-1-t)+t\right]=\frac{1}{t}\left[1-\left(\frac{1+t}{t}\right)^{p-1}\right]<0,
\end{aligned}
$$

and $q$ is monotone decreasing in $t$.

$$
q(1, p)=1-2\left[2^{p}-1\right] \sum_{j=1}^{\infty} \frac{1}{(j+1)^{p}} .
$$

The function in brackets is convex in $p$ for $p>1$. So also is the series. Therefore, so is the product. Multiplying by -1 yields a concave function. Since 1 is also concave, $q(1, p)$ is a concave function of $p$ for $p>1$. Since

$$
\lim _{p \rightarrow \infty} q(1, p)=0
$$

$g(0)$ is negative for those values of $t>t_{0}$, where $t_{0}$ satisfies (23); that is, (6) of Theorem 2, and $L^{p}=f(0)$.

The Rhaly $s$-Cesáro matrices [5] are factorable matrices with nonzero entries $a_{n}=$ $(n+1)^{-s}, s>1$, and each $b_{k}=1$. Thus $t_{n}=(n+1)^{s-1}$ and $y_{n}=n+1$.

Theorem 6. For the Rhaly s-Cesáro matrices, $L^{p}=f(\infty)$ for $p, s>1$. 
8 Factorable matrices

Proof. First, we will show that conditions (a)-(c) of Lemma 4 are satisfied,

$$
\begin{gathered}
v(r)=(r+1)^{p}, \quad v^{\prime}(r)=p(r+1)^{p-1}, \\
v^{\prime \prime}(r)=p(p-1)(r+1)^{p-2},
\end{gathered}
$$

and conditions (a) and (b) are satisfied,

$$
v^{\prime \prime \prime}(r)=p(p-1)(p-2)(r+1)^{p-3} .
$$

Therefore

$$
\begin{aligned}
2\left(v^{\prime \prime}\right)^{2}-v^{\prime} v^{\prime \prime \prime} & =2\left(p(p-1)(r+1)^{p-2}\right)^{2}-p(r+1)^{p-1} q(p-1)(p-2)(r+1)^{p-3} \\
& =p^{2}(p-1)(r+1)^{2 p-4}[2(p-1)-(p-2)] \\
& =p^{3}(p-1)(r+1)^{2 p-4}>0,
\end{aligned}
$$

and condition (c) is satisfied,

$$
0 \leq h(r) \leq \sum_{j=r+1}^{\infty} \frac{1}{(j+1)^{s p}}
$$

Therefore $\lim _{r \rightarrow \infty} h(r)=0$ and condition (5) of Theorem 2 is satisfied. Thus $g$ is monotone decreasing in $r$,

$$
g(r)=\sum_{j=0}^{r} \frac{1}{(j+1)^{(s-1) p}}+(r+1)(r+2)\left[(r+1)^{s-1}-(r+2)^{s-1}\right] \sum_{j=r+1}^{\infty} \frac{1}{(j+1)^{p s}} .
$$

It then follows that

$$
\lim _{r \rightarrow \infty} g(r)=\sum_{j=0}^{\infty} \frac{1}{(j+1)^{(p-1) s}}>0
$$

and so $L^{p}=f(\infty)$.

The Rhaly terraced matrices [6] are factorable matrices with each $b_{k}=1$ and $a_{n}=a_{n}$, where $\left\{a_{n}\right\}$ is a monotone decreasing positive sequence such that $\lim (n+1) a_{n}$ exists. Clearly $t_{n}=(n+1) a_{n}$ and $y_{n}=n+1$.

Theorem 7. For the Rhaly terraced matrices, $L^{p}=f(0)$ for $p>1$.

Proof. Since $y_{n}=n+1$, the first part of the proof of Theorem 6 applies and $h$ is monotone decreasing in $r$,

$$
h(r)=\frac{a_{r+1}^{p}\left[(r+2)^{p}-(r+3)^{p}\right]}{(r+1)^{p}-2(r+2)^{p}+(r+3)^{p}}+\sum_{j=r+1}^{\infty} a_{j}^{p} \leq \sum_{j=r+1}^{\infty} a_{j}^{p},
$$


and $\lim h(r)=0$, so that $g$ is monotone decreasing in $r$,

$$
\begin{aligned}
g(0) & =a_{0}^{p}+2\left[1-2^{p-1}\right] \sum_{j=1}^{\infty} a_{j}^{p} \\
& =a_{0}^{p}-2\left(2^{p-1}-1\right) a_{1}^{p}-2\left(2^{p-1}-1\right) \sum_{j=2}^{\infty} a_{j}^{p}<0,
\end{aligned}
$$

since $\left\{a_{n}\right\}$ is monotone decreasing. Therefore $L^{p}=f(0)$.

A weighted mean matrix is a factorable matrix with $a_{n}=1 / P_{n}, b_{k}=p_{k}$, where $\left\{p_{k}\right\}$ is a nonnegative sequence with $p_{0}>0$ and $P_{n}:=\sum_{k=0}^{n} p_{k}$.

Theorem 8. Let $\left(\bar{N}, p_{n}\right)$ be a weighted mean method with the $\left\{p_{n}\right\}$ nondecreasing. Then

$$
1+(r+1)\left(\frac{P_{r+1}}{P_{r}}\right)^{p}-(r+2)\left(\frac{P_{r+2}}{P_{r+1}}\right)^{p} \geq 0 \quad \text { for each } r \geq 0, p>1
$$

is a sufficient condition for $L^{p}=f(0)$.

Proof. Since a weighted mean matrix has row sums one, from (9),

$$
f(r)-f(r+1)=\frac{1}{r+2}+\Delta\left(\frac{P_{r}^{p}}{r+1}\right) \sum_{j=r+1}^{\infty} \frac{1}{P_{j}^{p}}
$$

Thus

$$
\begin{gathered}
g(r)=\frac{f(r)-f(r+1)}{\Delta\left(P_{r}^{p} /(r+1)\right)}=\frac{1}{(r+2) \Delta\left(P_{r}^{p} /(r+1)\right)}+\sum_{j=r+1}^{\infty} \frac{1}{P_{j}^{p}}, \\
g(r)-g(r+1)=\frac{1}{(r+2) \Delta\left(P_{r}^{p}(r+1)\right)}-\frac{1}{(r+3) \Delta\left(P_{r+1}^{p} /(r+2)\right)}+\frac{1}{P_{r+1}^{p}} \\
=\frac{1}{P_{r+1}^{p}(r+2)(r+3) \Delta\left(P_{r}^{p} /(r+1)\right) \Delta\left(P_{r+1}^{p} /(r+2)\right)} m(r),
\end{gathered}
$$

where

$$
\begin{aligned}
m(r)= & P_{r+1}^{p}(r+3) \Delta\left(\frac{P_{r+1}^{p}}{r+2}\right)-P_{r+1}^{p}(r+2) \Delta\left(\frac{P_{r}^{p}}{r+1}\right)+(r+2)(r+3) \Delta\left(\frac{P_{r}^{p}}{r+1}\right) \Delta\left(\frac{P_{r+1}^{p}}{r+2}\right) \\
= & P_{r+1}^{p}\left[(r+3)\left(\frac{P_{r+1}^{p}}{r+2}-\frac{P_{r+2}^{p}}{r+3}\right)-(r+2)\left(\frac{P_{r}^{p}}{r+1}-\frac{P_{r+1}^{p}}{r+2}\right)\right] \\
& +(r+2)(r+3)\left(\frac{P_{r}^{p}}{r+1}-\frac{P_{r+1}^{p}}{r+2}\right)\left(\frac{P_{r+1}^{p}}{r+2}-\frac{P_{r+2}^{p}}{r+3}\right)
\end{aligned}
$$




$$
\begin{aligned}
= & P_{r+1}^{p}\left[\left(\frac{r+3}{r+2}\right) P_{r+1}^{p}-P_{r+2}^{p}-\left(\frac{r+2}{r+1}\right) P_{r}^{p}+P_{r+1}^{p}\right] \\
& +\left(\frac{r+3}{r+1}\right) P_{r}^{p} P_{r+1}^{p}-\left(\frac{r+3}{r+2}\right)\left(P_{r+1}^{p}\right)^{2}-P_{r}^{p} P_{r+2}^{p}\left(\frac{r+2}{r+1}\right)+P_{r+1}^{p} P_{r+2}^{p} \\
= & P_{r+1}^{p}\left[\left(\frac{r+3}{r+2}\right) P_{r+1}^{p}-P_{r+2}^{p}-\left(\frac{r+2}{r+1}\right) P_{r}^{p}+P_{r+1}^{p}+\left(\frac{r+3}{r+1}\right) P_{r}^{p}-\left(\frac{r+3}{r+2}\right) P_{r+1}^{p}+P_{r+2}^{p}\right] \\
& -\left(\frac{r+2}{r+1}\right) P_{r}^{p} P_{r+2}^{p} \\
= & P_{r+1}^{p}\left[\frac{1}{r+1} P_{r}^{p}+P_{r+1}^{p}\right]-\left(\frac{r+2}{r+1}\right) P_{r}^{p} P_{r+2}^{p} \\
= & \frac{1}{r+1}\left[P_{r}^{p} P_{r+1}^{p}+(r+1)\left(P_{r+1}^{p}\right)^{2}-(r+2) P_{r}^{p} P_{r+2}^{p}\right] \\
= & \frac{P_{r}^{p} P_{r+1}^{p}}{r+1}\left[1+(r+1)\left(\frac{P_{r+1}}{P_{r}}\right)^{p}-(r+2)\left(\frac{P_{r+2}}{P_{r+1}}\right)^{p}\right] \geq 0,
\end{aligned}
$$

which is $\left[7\right.$, Theorem 1 , condition (1)] without any monotonicity condition on the $\left\{p_{n}\right\}$. Thus $g$ is monotone decreasing in $r$.

Since $\left\{p_{n}\right\}$ is nondecreasing, $P_{r} \leq(r+1) p_{r}$; that is, $p_{r} / P_{r} \geq(r+1)^{-1}$. Thus

$$
\frac{P_{r+1}}{P_{r}}=1+\frac{p_{r+1}}{P_{r}} \geq 1+\frac{p_{r}}{P_{r}} \geq \frac{r+2}{r+1},
$$

and $P_{r+1} / P_{r}(r+2) \geq P_{r}(r+1)$.

Using (44), since $p>1$,

$$
\begin{aligned}
\lim |g(r)| & =\lim \left|\frac{(r+1) P_{r}^{p}}{P_{r+1}^{p}\left[(r+2) P_{r}^{p}-(r+1) P_{r+1}^{p}\right]}\right| \\
& =\lim \left|\frac{r+1}{P_{r+1}^{p}\left[(r+2)-(r+1)\left(P_{r+1} / P_{r}\right)^{p}\right]}\right| \\
& =\lim \frac{(r+1)}{P_{r+1}^{p}\left[(r+1)\left(P_{r+1} / P_{r}\right)^{p}-(r+2)\right]} .
\end{aligned}
$$

Using the fact that $(1+x)^{p} \geq 1+p x$ for $p>1, x>-1$,

$$
\begin{aligned}
\lim |g(x)| & \leq \lim \frac{(r+1)}{P_{r+1}^{p}\left[(r+1)\left(1+p p_{r+1} / P_{r}\right)-(r+2)\right]} \\
& =\lim \frac{r+1}{P_{r}\left[p(r+1) p_{r+1} / P_{r}-1\right]} \\
& \leq \lim \frac{r+1}{P_{r}(p-1)}=0 .
\end{aligned}
$$


Therefore $\lim g(r)=0$ and $g$ is positive for all $r$. From (44), since $\Delta\left(P_{r}^{p} /(r+1)\right)<0$, $L^{p}=f(0)$.

Corollary 9. Suppose $(\bar{N}, p)$ is a weighted mean method with $p_{n}=(n+1)^{\alpha}, \alpha \geq 1$. Then $L^{p}=f(0)$.

Proof. To show that (42) is satisfied, it is sufficient to show that

$$
(r+1)\left(\frac{P_{r+1}}{P_{r}}\right)^{p}
$$

is convex. The function $r=1$ is trivially convex. Since $p>1$, it will be enough to show that $P_{r+1} / P_{r}$ is convex.

Define

$$
n(r)=1+\frac{p_{r+1}}{P_{r}}=1+\frac{(r+2)^{\alpha}}{\sum_{k=0}^{r}(k+1)^{\alpha}} .
$$

Then

$$
\begin{aligned}
\Delta^{2} n(r) & =\frac{(r+2)^{\alpha}}{\sum_{k=0}^{r}(k+1)^{\alpha}}-\frac{2(r+3)^{\alpha}}{\sum_{k=0}^{r+1}(k+1)^{\alpha}}+\frac{(r+4)^{\alpha}}{\sum_{k=0}^{r+2}(k+1)^{\alpha}} \\
& =\frac{n(r)}{\left(\sum_{k=0}^{r}(k+1)^{\alpha}\right)\left(\sum_{k=0}^{r+1}(k+1)^{\alpha}\right)\left(\sum_{k=0}^{r+2}(k+1)^{\alpha}\right)},
\end{aligned}
$$

where

$$
\begin{aligned}
n(r)= & (r+2)^{\alpha}\left(\sum_{k=0}^{r+1}(k+1)^{\alpha}\right)\left(\sum_{k=0}^{r+2}(k+1)^{\alpha}\right)-2(r+3)^{\alpha}\left(\sum_{k=0}^{r}(k+1)^{\alpha}\right)\left(\sum_{k=0}^{r+2}(k+1)^{\alpha}\right) \\
& +(r+4)^{\alpha}\left(\sum_{k=0}^{r}(k+1)^{\alpha}\right)\left(\sum_{k=0}^{r+1}(k+1)^{\alpha}\right) \\
= & (r+2)^{\alpha}\left[\sum_{k=0}^{r}(k+1)^{\alpha}+(r+2)^{\alpha}\right] \times\left[\sum_{k=0}^{r}(k+1)^{\alpha}+(r+2)^{\alpha}+(r+3)^{\alpha}\right] \\
& -2(r+3)^{\alpha}\left(\sum_{k=0}^{r}(k+1)^{\alpha}\right)\left(\sum_{k=0}^{r}(k+1)^{\alpha}+(r+2)^{\alpha}+(r+3)^{\alpha}\right) \\
& +(r+4)^{\alpha}\left(\sum_{k=0}^{r}(k+1)^{\alpha}\right)\left(\sum_{k=0}^{r}(k+1)^{\alpha}+(r+2)^{\alpha}\right) \\
= & \left(\sum_{k=0}^{r}(k+1)^{\alpha}\right)^{2}\left[(r+2)^{\alpha}-2(r+3)^{\alpha}+(r+4)^{\alpha}\right]
\end{aligned}
$$




$$
\begin{aligned}
& +\left[(r+2)^{2 \alpha}+(r+2)^{2 \alpha}+((r+2)(r+3))^{\alpha}\right. \\
& \left.\quad-2((r+2)(r+3))^{\alpha}-2(r+3)^{2 \alpha}+((r+4)(r+2))^{\alpha}\right] \\
& \quad \times\left(\sum_{k=0}^{r}(k+1)^{\alpha}\right)+(r+2)^{3 \alpha}+(r+2)^{2 \alpha}(r+3)^{\alpha} .
\end{aligned}
$$

Since $\alpha \geq 1,(r+2)^{\alpha}$ is convex, so the first quantity in brackets is positive. The second quantity in brackets can be written in the form

$$
(r+2)^{\alpha}\left\{(r+2)^{\alpha}-2(r+3)^{\alpha}+(r+4)^{\alpha}\right\}+(r+2)^{\alpha}+(r+3)^{\alpha}\left((r+2)^{\alpha}-2\right),
$$

which is positive. Therefore $n(r)$ is convex and (42) is satisfied.

Corollary 10. Let $1<p<\infty$, H the Hausdorff matrix generated by $\mu_{n}=a /(n+a), a \geq 1$. Then $L^{p}=f(0)$.

Proof. $H$ is also a weighted mean matrix with $p_{n}=p_{0} \Gamma(n+a) / \Gamma(a+1) \Gamma(n+1)$ and $P_{n}=$ $p_{0} \Gamma(n+a+1) / \Gamma(a+1) \Gamma(n+1)$. Substituting in (42), one obtains

$$
1+(r+1)\left(\frac{r+a+1}{r+1}\right)^{p}-(r+2)\left(\frac{r+a+2}{r+2}\right)^{p}
$$

and it is sufficient to prove that $(r+a+1) /(r+1)$ is convex, which it is.

The Cesáro matrix of order one, written $(C, 1)$, is a Hausdorff matrix with generating sequence $\mu_{n}=(n+1)^{-1}$.

Corollary 11. For $(C, 1), L^{p}=f(0)$.

Proof. Use Corollary 10 with $a=1$.

Remarks 12. (1) The condition that the $\left\{p_{n}\right\}$ be nondecreasing is not a necessary condition for $L^{p}=f(0)$. For example, take $p_{n}=2 /(n+1)(n+2)$. Then $\left\{p_{n}\right\}$ is monotone decreasing and satisfies (42) and

$$
\Delta\left(\frac{P_{r}}{r+1}\right)<0
$$

(2) Bennett [1] proved that $L^{p}=f(0)$ for the Hilbert matrix.

(3) In [2], Bennett has shown that $L^{p}=f(0)$ for each Hausdorff matrix $H \in B\left(\ell^{p}\right)$ with nonnegative entries.

(4) No results have been established for Nörlund matrices.

An interesting open question is the following. If $\left\{p_{n}\right\}$ is nondecreasing, must $\left\{p_{n}\right\}$ satisfy (42)? 


\section{References}

[1] G. Bennett, Lower bounds for matrices, Linear Algebra and Its Applications 82 (1986), 81-98.

[2] __ Lower bounds for matrices. II, Canadian Journal of Mathematics 44 (1992), no. 1, 5474.

[3] R. Lyons, A lower bound on the Cesàro operator, Proceedings of the American Mathematical Society 86 (1982), no. 4, 694.

[4] H. C. Rhaly Jr., Discrete generalized Cesàro operators, Proceedings of the American Mathematical Society 34 (1986), 225-232.

[5] _ _ p-Cesàro matrices, Houston Journal of Mathematics 15 (1989), no. 1, 137-146.

[6] _ Terraced matrices, The Bulletin of the London Mathematical Society 21 (1989), no. 4, 399-406.

[7] B. E. Rhoades, Lower bounds for some matrices, Linear and Multilinear Algebra 20 (1987), no. 4, $347-352$.

[8] _ Lower bounds for some matrices. II, Linear and Multilinear Algebra 26 (1990), no. 1-2, $49-58$.

B. E. Rhoades: Department of Mathematics, Indiana University, Bloomington, IN 47405-7106, USA E-mail address: rhoades@indiana.edu

Pali Sen: Department of Mathematics and Statistics, University of North Florida, Jacksonville, FL 32224, USA

E-mail address: psen@unf.edu 


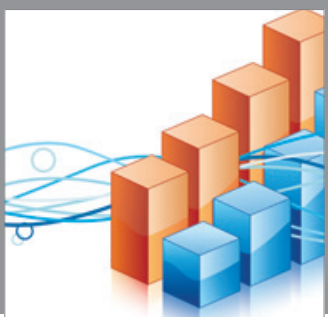

Advances in

Operations Research

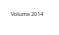

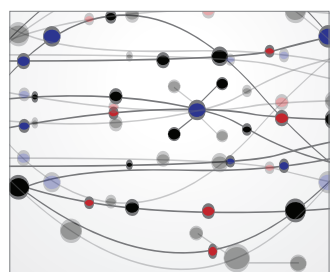

\section{The Scientific} World Journal
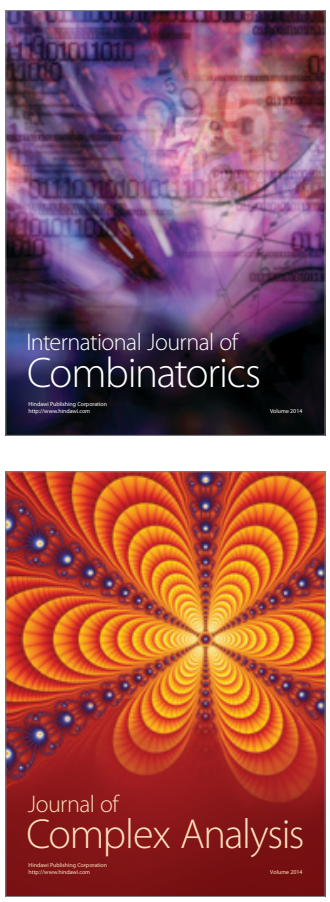

International Journal of

Mathematics and

Mathematical

Sciences
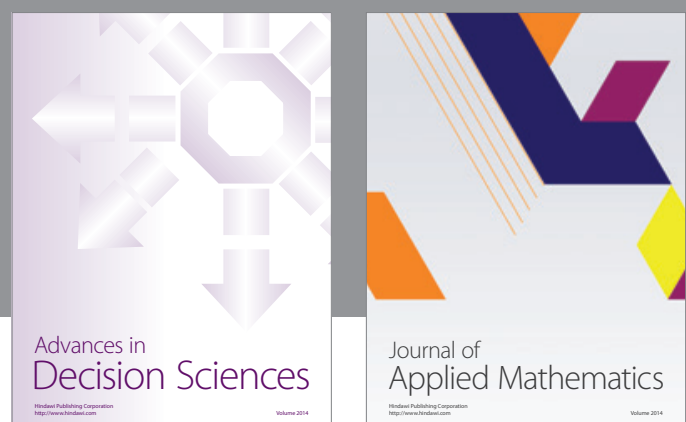

Journal of

Applied Mathematics
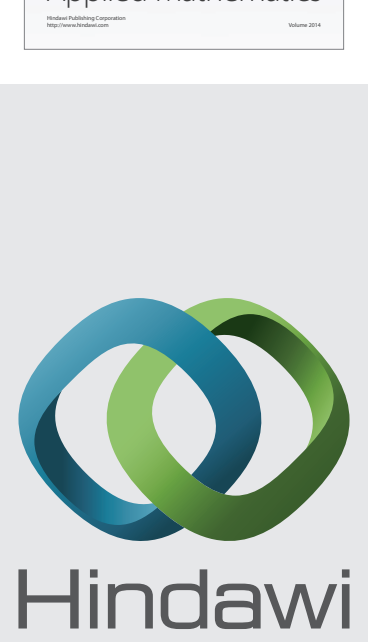

Submit your manuscripts at http://www.hindawi.com
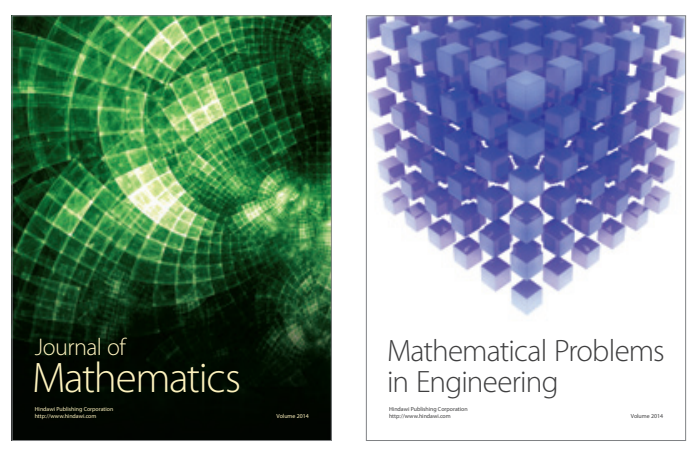

Mathematical Problems in Engineering
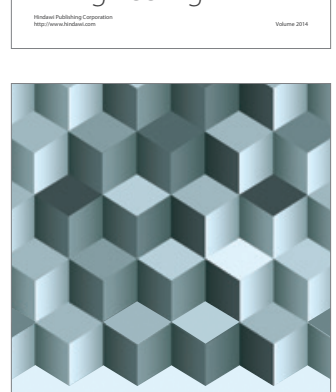

Journal of

Function Spaces
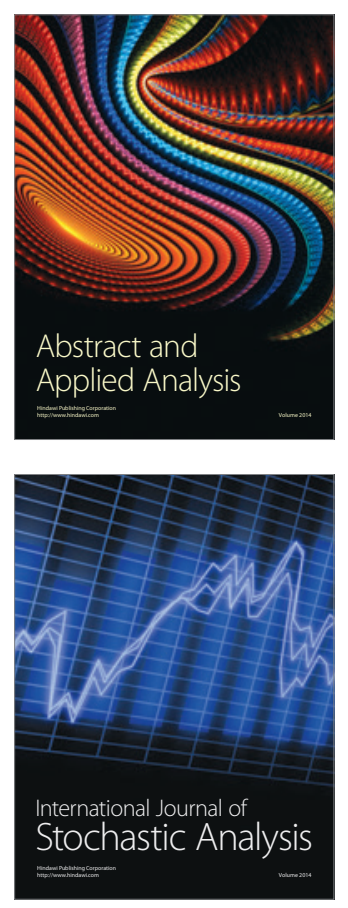

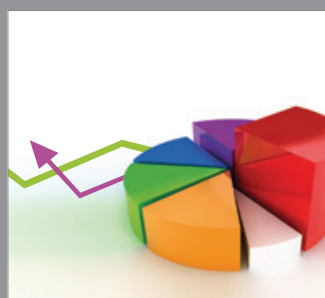

ournal of

Probability and Statistics

Promensencen
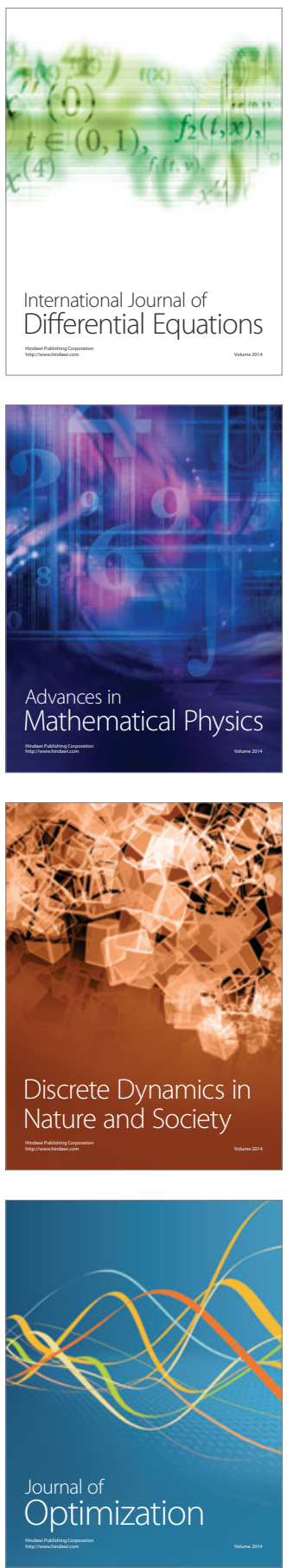